\title{
RXTE confirmation of the intermediate polar status of Swift J0732.5-1331
}

\author{
O. W. Butters ${ }^{1}$, E. J. Barlow ${ }^{1}$, A. J. Norton ${ }^{1}$, and K. Mukai ${ }^{2}$ \\ 1 Department of Physics and Astronomy, The Open University, Walton Hall, Milton Keynes MK7 6AA, UK \\ e-mail: o.w.butters@open.ac.uk \\ 2 CRESST and X-ray Astrophysics Laboratory NASA/GSFC, Greenbelt, MD 20771, USA; Department of Physics, \\ University of Maryland, Baltimore county, 1000 Hilltop Circle, Baltimore, MD 21250, USA
}

Accepted 18 September 2007 / Received 11 October 2007

\section{ABSTRACT}

\begin{abstract}
Aims. We intend to establish the X-ray properties of Swift J0732.5-1331 and therefore confirm its status as an intermediate polar. Methods. We analysed $36240 \mathrm{~s}$ of X-ray data from RXTE. Frequency analysis was used to constrain temporal variations and spectral analysis used to characterise the emission and absorption properties.

Results. The X-ray spin period is confirmed to be 512.4(3) s with a strong first harmonic. No modulation is detected at the candidate orbital period of $5.6 \mathrm{~h}$, but a coherent modulation is present at the candidate $11.3 \mathrm{~h}$ period. The spectrum is consistent with a $37 \mathrm{keV}$ bremsstrahlung continuum with an iron line at $6.4 \mathrm{keV}$ absorbed by an equivalent hydrogen column density of around $10^{22}$ atoms $\mathrm{cm}^{-2}$. Conclusions. Swift J0732-1331 is confirmed to be an intermediate polar.
\end{abstract}

Key words. stars: binaries: general - stars: novae, cataclysmic variables - stars: individual: Swift J0732.5-1331 - X-rays: binaries

\section{Introduction to magnetic cataclysmic variables}

Intermediate polars (IPs) are a sub-class of cataclysmic variables (CVs). They fill the phase space, in terms of magnetic field strength, and spin and orbital periods, between non-magnetic $\mathrm{CVs}$ and the strongly magnetic synchronously rotating polars. The magnetic field strength is believed to be in the range of a few MG to tens of MG at the white dwarf surface. This is large enough to dramatically alter the accretion flow, yet not large enough to synchronize the spin and orbital periods. This magnetic field gives rise to the defining characteristic of the subclass, that of X-ray variation pulsed at the spin period of the white dwarf. For an exhaustive review of CVs see e.g. Warner (1995).

There are between twenty six ${ }^{1}$ and fifty IPs currently known (depending on the selection criteria used). The hard X-ray selected object, Swift J0732.5-1331 (hereafter J0732), is a suspected IP in need of confirmation. The circumstance of its discovery makes J0732 similar to the host of candidate IPs that have been discovered to be powerful emitters of hard X-rays/soft gamma-rays in the 20-100 keV range in the INTEGRAL/IBIS survey (Barlow et al. 2006). We have embarked on a campaign of pointed RXTE observations of these hard X-ray discovered candidate IPs. Here we present the first results of our campaign on J0732.

\section{Previous observations of Swift J0732.5-1331}

There is to date no peer-reviewed analysis of J0732 published in the literature. There are however several mentions of it in Astronomical Telegrams, which we summarise below. These

\footnotetext{
${ }^{1}$ http://asd.gsfc.nasa.gov/Koji.Mukai/iphome/ iphome.html as of 23/8/7.
}

were all published over the course of a couple of months in early 2006.

J0732 was first detected by Ajello et al. (2006) with the Swift Burst Alert Telescope and Swift X-ray telescope (XRT). With the XRT 600 counts in $3400 \mathrm{~s}$ were recorded, coincident with the ROSAT source 1RXS J073237.6-133113. This is also coincident with the 2MASS source J073237.64-133109.4, a proposed K main-sequence star 400 pc away. Based on its X-ray luminosity and colours, Ajello et al. (2006) suggested a CV identification for the object.

Masetti et al. (2006) used the BFOSC instrument on the G.D. Cassini $1.5 \mathrm{~m}$ telescope to obtain the optical spectrum of the counterpart to J0732. Two objects were found close to the reported position, a normal G/K type Galactic star (the 2MASS source) and a fainter one deemed to be the true optical counterpart. The spectral signature of the system was concluded to be that of an accretion disc in a low mass X-ray binary.

Patterson et al. (2006) also obtained low resolution spectra, this time on the MDM $2.4 \mathrm{~m}$ telescope, of the 2MASS optical counterpart proposed by Ajello et al. (2006) (i.e. the field star), concluding that it was indeed a normal $\mathrm{G}$ star. In the same telegram Patterson et al. (2006) also reported optical photometry (obtained by the small telescope network of the Center for Backyard Astrophysics ${ }^{2}$ ) which revealed a stable pulsation period of 512.42(3) s with most of the power in the first harmonic. This was deemed to be the spin period of a rotating white dwarf and Patterson et al. (2006) consequently suggested an IP classification for the object. A possible $11.3 \mathrm{~h}$ orbital signal was also suggested, but owing to its low amplitude, this required the binary to be close to face on, as any variation in brightness due to the projected Roche lobe filling secondary was small.

\footnotetext{
${ }^{2}$ http://cba.phys. columbia.edu/
} 


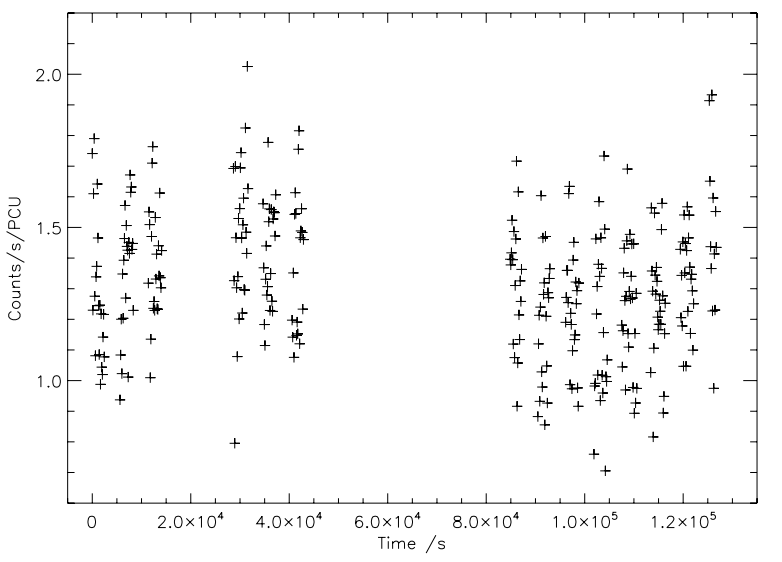

Fig. 1. 2-10 keV background subtracted light curve of J0732.5-1331. The zero time corresponds to the start of the observations at JD 2454295.30035 . The data is binned into bins of $128 \mathrm{~s}$ width. The typical error on each point is \pm 0.2 .

Marsh et al. (2006) subsequently used ULTRACAM mounted on the William Herschel Telescope to observe the optical counterpart of J0732. The spin pulsation detected by Patterson et al. (2006) was seen. The counterpart and the non-associated field star were found to be approximately $1.8^{\prime \prime}$ apart.

Torres et al. (2006) performed spectral analysis of the optical counterpart at the Mt. Hopkins $1.5 \mathrm{~m}$ telescope. Balmer emission lines from $\mathrm{H} \alpha$ to at least $\mathrm{H} \gamma$ were found. This reinforced its classification as a probable IP. However, despite seeing a variation in the radial velocities of the various emission lines, they were unable to determine an orbital period.

Wheatley et al. (2006) later reanalysed the original Swift data reported in Ajello et al. (2006) and found an X-ray pulsation at the proposed spin period. The modulation was found to be single peaked and only present below $2 \mathrm{keV}$. Reanalysis of the spectral data suggested a temperature typical of intermediate polars $(k T \sim 20 \mathrm{keV})$. This data set is, however, short and suffers from severe aliasing effects.

Thorstensen et al. (2006) carried out time series spectroscopy at the MDM Observatory on the optical counterpart. The radial velocities of the $\mathrm{H} \alpha$ emission lines were found to vary periodically with a period of $0.2335(8)$ days $(5.60(2) \mathrm{h}$ ), which was interpreted as the orbital period of the system. We note this is close to half the photometric period suggested by Patterson et al. (2006).

Given all this information, J0732 is strongly suspected to be an intermediate polar, but the only way to confirm this is the unambiguous detection of pulsed hard X-ray emission at the spin period.

\section{Observations and data reduction}

Data were obtained from the RXTE satellite (Bradt et al. 1993) with the PCA instrument over two consecutive days, from 13 th-15th July 2007. The total time on target was $36240 \mathrm{~s}$, comprising fourteen approximately equal segments of one satellite orbit each. Initial data reduction was done with the standard FTOOLS, and the flux was normalised according to the number of correctly functioning PCUs. For the light curve analysis PCUs 2, 3 , and 4 were used; whilst for the spectral analysis only PCU 2 was used as the other PCUs were only turned on infrequently. Only the top layer of each PCU was included in the measurements and the time resolution of the data was $16 \mathrm{~s}$. Background subtracted light curves were constructed in four energy

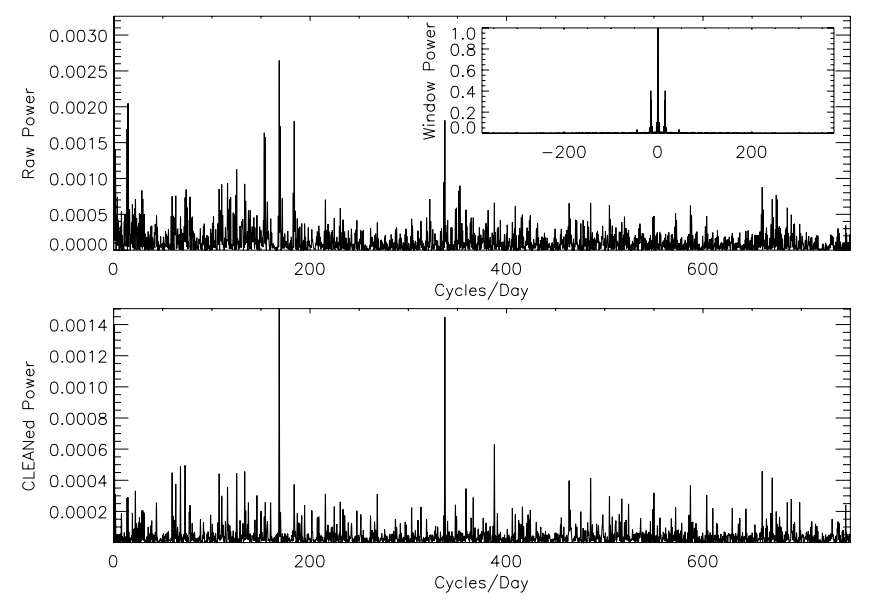

Fig. 2. CLEANed periodogram. The upper plot shows the raw periodogram, with the window function inset; the lower plot shows the deconvolved (CLEANed) periodogram.

Table 1. Modulation depths of the pulse profile in different energy bands. Modulation depth is defined here as the semi-amplitude of a fitted sinusoid divided by the fitted mean.

\begin{tabular}{cccc}
\hline \hline $\begin{array}{c}\text { Energy band } \\
(\mathrm{keV})\end{array}$ & $\begin{array}{c}\text { Modulation depth } \\
(\%)\end{array}$ & $\begin{array}{c}\text { Uncertainty } \\
(\%)\end{array}$ & $\begin{array}{c}\text { Fitted mean } \\
\left(\mathrm{ct} \mathrm{s}^{-1} \mathrm{PCU}^{-1}\right)\end{array}$ \\
\hline $2-10$ & 8 & 1 & 1.31 \\
$2-4$ & 16 & 3 & 0.25 \\
$4-6$ & 7 & 2 & 0.46 \\
$6-10$ & 7 & 2 & 0.56 \\
$10-20$ & 10 & 4 & 0.28 \\
\hline
\end{tabular}

bands: $2-4 \mathrm{keV}, 4-6 \mathrm{keV}, 6-10 \mathrm{keV}$ and $10-20 \mathrm{keV}$, as well as a combined 2-10 keV band for maximum signal-to-noise. A mean X-ray spectrum was also extracted.

\subsection{Light curve analysis}

The raw count rate varied between 3.9 and 5.4 count $\mathrm{s}^{-1} \mathrm{PCU}^{-1}$. The background count rate, generated from the calibration files, varied between 2.9 and 3.8 count $\mathrm{s}^{-1} \mathrm{PCU}^{-1}$. The background subtracted 2-10 keV light curve is shown in Fig. 1. The data were subsequently analysed with a variable gain implementation of the CLEAN algorithm (Lehto 1997) to discover any periodicities and discount any aliasing effects. The results of this are shown in Fig. 2.

\subsubsection{Spin period}

Strong peaks are evident in the CLEANed periodogram at $168 \mathrm{cy}-$ cles day $^{-1}$ (512 s) and at its first harmonic, in the $2-10 \mathrm{keV}$ energy band. Similar signals are seen in each energy band. Analysis of the peaks yields a pulsation period of 512.4(3) s. The data in each of the energy resolved light curves were then folded at the $512.4 \mathrm{~s}$ period, and Fig. 3 shows the result in the $2-10 \mathrm{keV}$ energy band. The modulation depths of the pulse profile were then estimated by fitting a sinusoid to the folded data in each energy band and dividing the semi-amplitude by the fitted mean. The results of this are shown in Table 1.

\subsubsection{Orbital period}

The windowing of the data is such that no reliable signal can be extracted for periods of a few hours from the periodogram, 


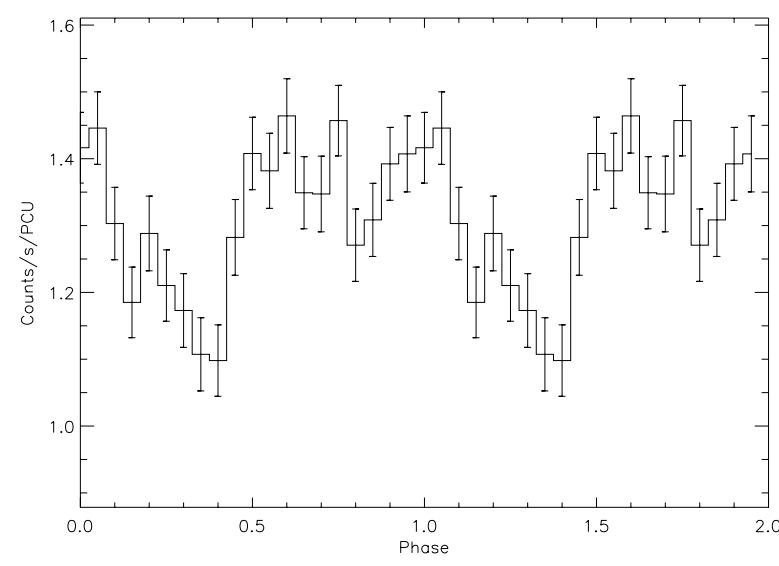

Fig. 3. 2-10 keV light curve folded at the $512.4 \mathrm{~s}$ pulse period with an arbitrary zero point. Two cycles are shown for clarity.

therefore no reliable orbital period can be found. Folding the $\mathrm{X}$-ray data at the $5.6 \mathrm{~h}$ spectroscopic period (Thorstensen et al. 2006) yields no coherent modulation, but folding it at the $11.3 \mathrm{~h}$ photometric period suggested by Patterson et al. (2006) gives a plausible signal (see Fig. 4).

\subsection{Spectral analysis}

Analysis of the X-ray spectrum was carried out with the XSPEC package. The best fit for a simple photoelectrically absorbed bremsstrahlung model had the parameters $k T=37 \pm$ $7 \mathrm{keV}$ and $n_{\mathrm{H}}=(2.0 \pm 0.5) \times 10^{22} \mathrm{~cm}^{-2}$ (reduced $\left.\chi^{2}=3.0\right)$. The residuals of this plot indicate the presence of an excess at approximately $6.5 \mathrm{keV}$. Keeping the temperature and column density fixed and fitting a Gaussian to this region indicates an iron line at $6.4 \pm 0.1 \mathrm{keV}$ with a width of $\sigma=0.3 \pm 0.1 \mathrm{keV}$ (reduced $\chi^{2}=1.0$ ), as shown in Fig. 5 .

\section{Discussion}

The strong X-ray signal at the 512.4(3) s pulse period seen at all energies is characteristic of IPs and confirms the nature of the object. Patterson et al. (2006) found a much stronger peak at the first harmonic in their frequency analysis of the optical photometry data. This is characteristic of a double-peaked pulse profile and indicates that two emission regions can be seen. The X-ray data reported here exhibit the same periodicity, but a somewhat different profile. The first harmonic in the X-ray data is still present, and the pulse profile consequently shows a second minimum superimposed on the pulse maximum, but the overall profile is only marginally double-peaked. The most likely geometry of this system is therefore such that one magnetic pole can always be seen, the other being behind the WD for most of the cycle. If the heights of the accretion columns are such that a fraction of the hidden pole's column can be seen at certain phases then the X-ray profile may be explained. If the optical emission arises from reprocessed X-rays (i.e. further up the accretion column) then it may be seen from both poles and this would explain the optical signal of Patterson et al. (2006).

The modulation depth of the X-ray pulse profile is approximately constant above $4 \mathrm{keV}$, implying that the dominant effect shaping the profile is geometric, probably self-occultation by the white dwarf. At the lowest energies $(2-4 \mathrm{keV})$ the modulation depth is higher, which implies that phase-varying photoelectric absorption (as well as occultation) is the process which causes the modulation. The spectral fitting also indicates the presence

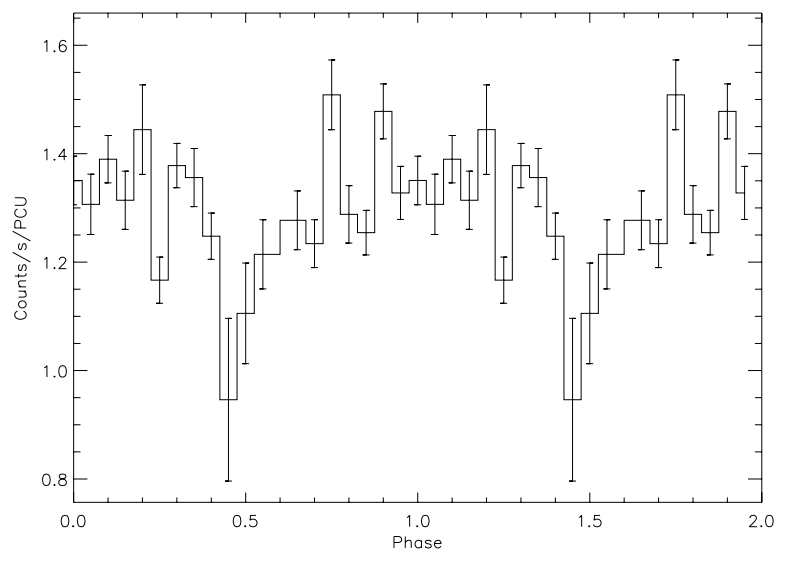

Fig. 4. 2-10 keV light curve folded at the $11.3 \mathrm{~h}$ photometric period suggested by Patterson et al. (2006) with an arbitrary zero point. Two cycles are shown for clarity.

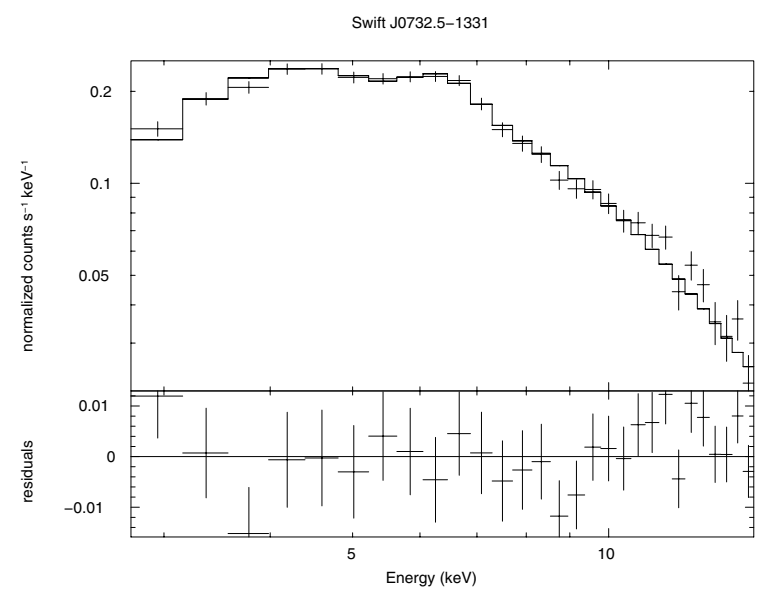

Fig. 5. 2.5-15 keV mean spectrum fitted with a photoelectrically absorbed bremsstrahlung plus iron line profile. Fitting parameters are $k T=37 \pm 7 \mathrm{keV}, n_{\mathrm{H}}=(2.0 \pm 0.5) \times 10^{22} \mathrm{~cm}^{-2}$, iron line fitted with a Gaussian centred on $6.4 \pm 0.1 \mathrm{keV}$ with $\sigma=0.3 \pm 0.1 \mathrm{keV}$. Reduced $\chi^{2}=1.0$.

of a significant local absorbing column, and has parameters that are typical of other IPs.

There is still some ambiguity about the orbital period of this system. The chance of it being the $11.3 \mathrm{~h}$ period suggested by Patterson et al. (2006) is now increased, given the X-ray signal seen here, but the possibility of aliasing in this data set means that it cannot be definitively said to be so and we cannot rule out the $5.6 \mathrm{~h}$ spectroscopic period found by Thorstensen et al. (2006). The absence of a beat period in the frequency analysis of the optical data does suggest that the true orbital period may be long, since no reprocessed radiation is seen from the face of the secondary star and therefore the bodies are likely to be far apart.

The lack of an X-ray beat signal in these RXTE data indicates that there is no significant stream-fed component to the flow. This suggests a relatively weak magnetic field strength and is consistent with the small $P_{\text {spin }} / P_{\text {orb }}$ ratio of the system (Norton et al. 2004), namely 0.025 or 0.013 depending on which is the correct orbital period.

The high temperature bremsstrahlung continuum and the presence of an iron line at $6.4 \mathrm{keV}$ reinforce the case for $\mathrm{J} 0732$ to be an IP since these are both features often seen in other IPs (Hellier \& Mukai 2004). 
Finally, we note that the average RXTE count rate ( $1.3 \mathrm{ct} \mathrm{s}^{-1} \mathrm{PCU}^{-1}$ in the $2-10 \mathrm{keV}$ band) is consistent ${ }^{3}$ with the value obtained with the Swift satellite, indicating that the system has not changed significantly in brightness since its discovery.

\section{Conclusion}

The unambiguous X-ray spin period detection at 512.4(3) s, along with the spectral fit to an absorbed $37 \mathrm{keV}$ bremsstrahlung model with an iron line, confirm the intermediate polar status of Swift J0732.5-1331. We are unable to determine the orbital period from these RXTE data although there is some indication of modulation at the previously suggested photometric period of $11.3 \mathrm{~h}$ and none at the spectroscopic period of $5.6 \mathrm{~h}$. To conclude we note that this system is similar, in terms of its small $P_{\text {spin }} / P_{\text {orb }}$ value, to the IPs RX J2133.7+5107 and NY Lup (IGR J15479-4529). Both of these are INTEGRAL hard X-ray sources and both also have soft X-ray components. We might therefore expect that Swift J0732.5-1331 would also display such characteristics upon further study.

\section{References}

Ajello, M., Greiner, J., Rau, A., et al. 2006, The Astronomer's Telegram, 697, 1 Barlow, E. J., Knigge, C., Bird, A. J., et al. 2006, MNRAS, 372, 224 Bradt, H. V., Rothschild, R. E., \& Swank, J. H. 1993, A\&AS, 97, 355 Hellier, C., \& Mukai, K. 2004, MNRAS, 352, 1037

Lehto, H. J. 1997, in Applications of time series analysis in astronomy and meteorology, ed. T. Subba Rao, M. B. Priestley, \& O. Lessi (London Chapman and Hall)

Marsh, T., Littlefair, S., \& Dhillon, V. 2006, The Astronomer's Telegram, 760, 1 Masetti, N., Bassani, L., Dean, A. J., Ubertini, P., \& Walter, R. 2006, The Astronomer's Telegram, 735, 1

Norton, A. J., Wynn, G. A., \& Somerscales, R. V. 2004, ApJ, 614, 349

Patterson, J., Halpern, J., Mirabal, N., et al. 2006, The Astronomer's Telegram, 757,1

Thorstensen, J. R., Patterson, J., Halpern, J., \& Mirabal, N. 2006, The Astronomer's Telegram, 767, 1

Torres, M. A. P., Steeghs, D., Garcia, M. R., et al. 2006, The Astronomer's Telegram, 763, 1

Warner, B. 1995, Cataclysmic variable stars, Cambridge Astrophysics Series (Cambridge, New York: Cambridge University Press)

Wheatley, P. J., Marsh, T. R., \& Clarkson, W. 2006, The Astronomer's Telegram, 765,1

${ }^{3}$ Using webpimms:

http://heasarc.gsfc.nasa.gov/Tools/w3pimms.html 